neter Weg (S. 293). Jesse empfiehlt daher wie auch Bergsdorf die inhaltliche Auseinandersetzung mit den Zielsetzungen der NPD. Nur so „erfährt der Bürger, was an den Parolen aus der Sicht demokratischer Kräfte nicht stimmt" (S. 294). Verbote rechtsextremistischer Vereine oder Parteien aber, so auch der Tenor im Beitrag von Julia Gerlach, haben den Fortbestand rechtsextremer Geisteshaltungen mittelfristig nicht verhindert. Im Gegenteil: „Was kurz nach der Vereinigung von kleinen Splittergruppen, Politsekten propagiert wird, verkündet heute eine bundesweit aktive Partei unter der besonderen Gunst des Grundgesetzes." (S. 258 f.)

Abschließend geht es im Sammelband um die Ideologie der NPD. Uwe Backes arbeitet das Profil der Partei im europäischen Vergleich heraus; Steffen Kailitz und Marc Brandstetter vertiefen die nationalsozialistische Ideologie der NPD. Brandstetter analysiert Parteiprogramme sowie Artikel des „Politischen Lexikons der NPD“ und der Deutschen Stimme. Hier, so der Verfasser, würde sich die NPD deutlich aggressiver als in offiziellen Dokumenten äußern und gäbe so ihren wahren Charakter preis (S. 317). Brandstetter zeichnet die programmatische Entwicklung der NPD vom Gründungsmanifest von 1964 über das Wertheimer Manifest von 1970 bis zum Düsseldorfer Programm nach, deckt zahlreiche Mythen des rechtsextremen Denkens auf und zeigt, dass die NPD nicht vollständig dem neonazistischen Spektrum zugerechnet werden kann, da hierzu wichtige Ideologieelemente fehlten (S. 335) - eine Tatsache, die seiner Meinung nach jedoch nicht verbergen kann, dass „die bürgerlich-konservativen Ideologiefragmente der Gründungs- und Frühphase weitgehend von nationalsozialistischen Fragmenten abgelöst worden sind“ (S. 335). Kailitz geht einen Schritt weiter. Insbesondere in Bezug auf die Sozialpolitik sieht er deutliche Parallelen zwischen den Forderungen der NSDAP und der NPD. „Wie die historische NSDAP“, so bemerkt er, „versucht die NPD, mit einem opulenten Programm an Versorgungsleistungen die Zustimmung der Deutschen zu gewinnen“ (S. 348).

Der von Backes und Steglich herausgegebene Band besticht vor allem durch den analytischen Tiefgang und die hohe Aktualität seiner Beiträge. Der Zuschnitt der Analyse erlaubt naturgemäß die Bearbeitung eines deutlich breiteren inhaltlichen Feldes. Dies kann und will die Monographie von Bergsdorf nicht leisten. Beide Publikationen vermitteln eine Vielzahl von Informationen, zeigen ein hohes Abstraktionsniveau und versprechen dem Leser daher gerade vor dem Hintergrund der derzeitigen politischen Debatte zur NPD einen hohen Nutzen.

Sebastian Rehse

\title{
Die NPD in Fragen und Antworten: hohe Aktualität
}

Virchow, Fabian und Christian Dornbusch (Hrsg.): 88 Fragen und Antworten zur NPD. Weltanschauung, Strategie und Auftreten einer Rechtspartei - und was Demokraten dagegen tun können, Wochenschau Verlag, Schwalbach/Ts. 2008, 336 Seiten, € 24,80.

Die Herausgeber Fabian Virchow und Christian Dornbusch stellen 88 Fragen zur NPD, die von insgesamt 45 Autoren in zwölf Kapiteln beantwortet werden. Ziel des Bandes ist es, „den aktuellen Stand der Forschung und seriöser journalistischer Recherche in einer Form zugänglich zu machen, die auch dem nicht wissenschaftlich geschulten Publikum einen 
qualifizierten Zugang zum Thema NPD ermöglicht“ (S. 13). Um es vorweg zu nehmen: dieses Ziel wird nur teilweise erreicht.

Sehr positiv ist zu vermerken, dass mit den 88 Fragen die NPD, als zur Zeit zentrale Akteurin im deutschen Rechtsextremismus, hinsichtlich ihrer Geschichte, ihrer Ideologie und ihrer Strategie umfassend erklärt und analysiert wird. Dazu werden - auch dies ist sehr zu begrüßen - eine Reihe von Primärquellen herangezogen und zitiert, die aber leider nicht immer durchgängig belegt sind. Somit vermittelt der Band eine aktuelle, realistische Einschätzung der Partei ohne den leider oft üblichen Alarmismus, vor allem hinsichtlich ihrer intellektuellen Kapazität. Hier heißt es zutreffend: „Von einer Intellektualisierung der NPD kann höchstens im Vergleich zum früheren Status quo der Partei gesprochen werden." (S. 93) Die Beschreibung Reiner Beckers, die NPD versuche sich als „Ort sozialer Wertschätzung" (S. 102) im Sinne Axel Honneths zu etablieren, trifft einerseits den Nagel analytisch auf den Kopf, zeigt aber andererseits die Schwäche des Bandes auf. Er ist eben doch eine zu schwere Kost für ein „nicht wissenschaftlich geschultes“ Publikum und eignet sich auch nicht als Nachschlagewerk. Schmerzlich vermisst wird hierzu zum Beispiel ein Schlagwortregister.

So werden die „88 Fragen“ wohl hauptsächlich von den „üblichen Verdächtigen“ der Rechtsextremismusforschung und den zivilgesellschaftlichen Akteuren der entsprechenden Bundes- und Landesprogramme rezipiert werden. Doch auch für diesen Personenkreis bietet der Band eine ganze Reihe von interessanten Informationen und Positionierungen. Neben den zuweilen recht launig geschriebenen Profilen des NPD-Führungspersonals („Ist Holger Apfel so harmlos, wie er aussieht?") gibt es sehr lesenswerte Texte unter anderem von Wolfgang Gessenharter („Haben nicht auch Neonazis ein Recht auf Meinungsfreiheit?“) und Peter Reif-Spirek („Was heißt eigentlich , die argumentative Auseinandersetzung in der Öffentlichkeit suchen'?"). Auch die Beiträge von Benno Hafeneger zur sozialen und zur Bildungsarbeit sind sehr erhellend; das Grundprinzip des Ansetzens „an den Problemen, die die Jugendlichen haben, nicht an den Problemen, die sie machen“ (S. 288), gehört ebenso ins Stammbuch aller geschrieben, die sich mit Rechtsextremismusprävention beschäftigen, wie das Diktum von Albert Scherr, „für demokratische und menschenrechtliche Prinzipien mit genuin demokratischen Mitteln einzutreten“ (S. 294). Während Scherr in diesem Sinne ein NPD-Parteiverbotsverfahren ablehnt, wird dieses von Virchow einige Seiten zuvor vehement gefordert.

Insgesamt dominiert allerdings die Erkenntnis, dass eine nachhaltige Auseinandersetzung mit dem Rechtsextremismus im Allgemeinen und der NPD im Besonderen nicht durch ein Mehr an Verboten oder Gesetzesverschärfungen (und schon gar nicht durch Gewalt auf der Straße), sondern nur durch entschlossenes Entgegentreten von Staat und Bürgern sowie aktives Widersprechen auf allen Ebenen erfolgen kann. Wie Hans-Gerd Jaschke betont, sollen Polizei und Justiz hier nur das „letzte Mittel gegen den Rechtsextremismus“ sein: „Vorrang hat die politische Auseinandersetzung.“ (S. 314) Und diese wird durch den Band von Virchow und Dornbusch eindeutig bereichert. 